\title{
The clinical features of spondyloepiphyseal dysplasia congenita resulting from the substitution of glycine 997 by serine in the $\alpha 1$ (II) chain of type II collagen
}

\author{
W G Cole, R K Hall, J G Rogers
}

\begin{abstract}
The features of a child with spondyloepiphyseal dysplasia congenita resulting from a mutation in one COL2A1 allele were studied. The child was heterozygous for a $G$ to $A$ transition in exon 48 that resulted in the substitution of glycine 997 by serine in the triple helical domain of $\alpha 1$ (II) chains of type II collagen. Her longitudinal growth was close to the mean growth curve for children with this chondrodysplasia. Expression of the mutation by chondrocytes would account for the abnormal growth and development of the bones of the limbs and spine. Early expression of the mutation by epithelial cells and later expression by chondrocytes of the developing craniofacial structures would also account for her complex pattern of craniofacial anomalies. The findings in this study confirm that mutations of exon 48 of the COL2A1 gene, that alter the normal Gly-X-Y triplet structure of the corresponding region of $\alpha 1$ (II) chains of type II collagen, produce the spondyloepiphyseal dysplasia congenita phenotype.

( $\mathcal{f}$ Med Genet 1993;30:27-35)
\end{abstract}

Department of

Paediatrics,

University of

Melbourne, Parkville,

Victoria 3052,

Australia.

W G Cole

Department of

Dentistry, Royal

Children's Hospital,

Parkville, Victoria

3052, Australia.

R K Hall

Department of Clinical Genetics,

Royal Children's

Hospital, Parkville,

Victoria 3052,

Australia.

J G Rogers

Correspondence to Professor Cole.

Received 26 June 1992 Accepted 13 July 1992
The COL2A1 gene encodes the $\alpha 1$ (II) chains of type II collagen which is found in hyaline cartilages, fibrocartilages, nucleus pulposis, and vitreous humour. ${ }^{1}$ Mutations of this gene have been identified in a family of chondrodysplasias characterised by abnormalities of these tissues. ${ }^{2}$ The family includes spondyloepiphyseal dysplasia congenita, achondrogenesis type II, hypochondrogenesis, some patients with Stickler syndrome, and Kniest dysplasia. The diversity of phenotypes in this family provides an opportunity to examine the relationships between the genotypes and phenotypes.

Heterozygous mutations of the COL2A1 gene have been identified in patients with spondyloepiphyseal dysplasia congenita. In one of them, there was a heterozygous deletion of $390 \mathrm{bp}$ from the middle of intron 47 to the splice donor site of intron $48 .^{3}$ The deletion eliminated exon 48 which normally encodes 36 amino acids from glycine 964 to alanine 999 of the triple helix of $\alpha 1$ (II) chains. In another patient, there was a tandem duplication of $45 \mathrm{bp}$ within exon 48 of one allele that would be expected to add 15 amino acids to the triple helical domain of the mutant $\alpha 1$ (II) chains. ${ }^{4}$ In contrast, a relatively mild form of autosomal dominant spondyloepiphyseal dysplasia, with the onset of symptoms of osteoarthritis in the second and third decades of life, was shown to be the result of a heterozygous substitution of arginine 519 by cysteine in the triple helix of $\alpha 1$ (II) chains. ${ }^{5}$ This defect was produced by a $\mathrm{C}$ to $\mathrm{T}$ transition in exon 31 .

Abnormal type II collagen has also been identified in achondrogenesis type II and hypochondrogenesis. In one case, a heterozygous point mutation in exon 46 converted GGC for glycine 943 in the triple helix to AGC for serine. ${ }^{6}$

Linkage has also been reported between restriction fragment length polymorphisms of the COL2Al gene and Stickler syndrome in some families. ${ }^{7}$ In one such family, a point mutation in exon 39 converted the codon CGA for arginine 732 of the triple helix of $\alpha$ (II) chains to TGA, a stop codon. ${ }^{8}$

Type II collagen anomalies have also been found in patients with Kniest dysplasia. In epiphyseal and physeal cartilages, the collagen fibrils are abnormal and the amount of the carboxy-propeptide of the pro $\alpha$ l(II) chains is reduced. ${ }^{9}$ Abnormal processing of the carboxy-propeptide has been proposed to account for these findings but the molecular defect is unknown. 9

In this paper, we describe the phenotypic consequences of a COL2A1 mutation in a child with typical spondyloepiphyseal dysplasia congenita. ${ }^{10}$ The low basal rate of transcription ('illegitimate transcription'), splicing, and polyadenylation of $\alpha 1$ (II) mRNA by cultured dermal fibroblasts and lymphoblastoid cells provided the opportunity to localise and sequence the mutation in amplified cDNA from this patient from whom affected tissue was unavailable. A sequence mismatch was identified using chemical modification of cDNA:cDNA heteroduplexes by hydroxylamine and cleavage with piperidine. The 
amplification products containing the mismatched region were sequenced and the mutation was shown to change the codon GGC for glycine 997 to AGC for serine in the triple helical domain of the $\alpha$ (II) chains. The corresponding region of the genomic DNA was sequenced and the heterozygous point mutation was shown to be in exon 48 of the COL2A1 gene. Allelic restriction mapping showed that neither parent carried the mutation in their leucocytes.

\section{Case report}

The girl was the third child of healthy, unrelated parents. Her sibs were normal and there was no family history of short stature. After a normal pregnancy of 40 weeks' duration, she was born by a breech vaginal delivery. She was well at birth but was noted to be small.

At birth, her length was $44 \mathrm{~cm}$, which was well below the 10th centile, while her weight of $2570 \mathrm{~g}$ and head circumference of $33.5 \mathrm{~cm}$ were within the low normal ranges. ${ }^{11}$ Her head was large relative to her short trunk and limbs, her face was flat, and the mandible was small with retrognathia. The limbs and spine were well aligned but her chest was short and broad. She had a $U$ shaped cleft palate involving the soft and hard palate.

Radiographs showed the typical features of spondyloepiphyseal dysplasia congenita (figs 1 to 3). ${ }^{2}$ The long bones of the upper and lower limbs were short, their metaphyses flared and epiphyses unossified. The ossification centres in the carpal and tarsal bones were smaller than normal. The pubic symphysis was not ossified and the ilia were short and broad. The bodies of the cervical and thoracic vertebrae were flatter than normal while they were ovoid

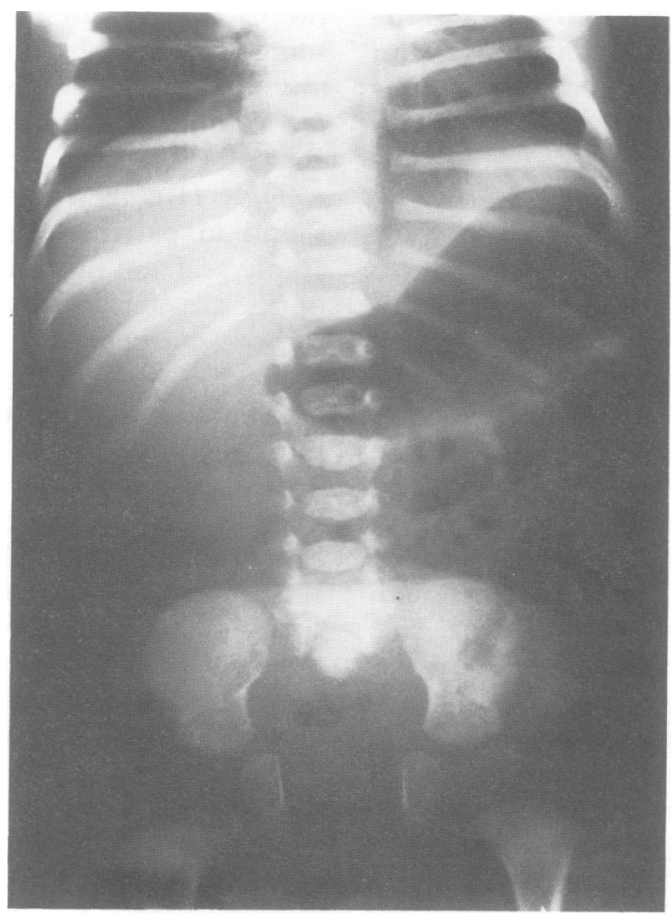

Figure 1 Radiograph of the trunk at 1 week of age showing generalised platyspondyly and absent ossification of the pubic symphysis.

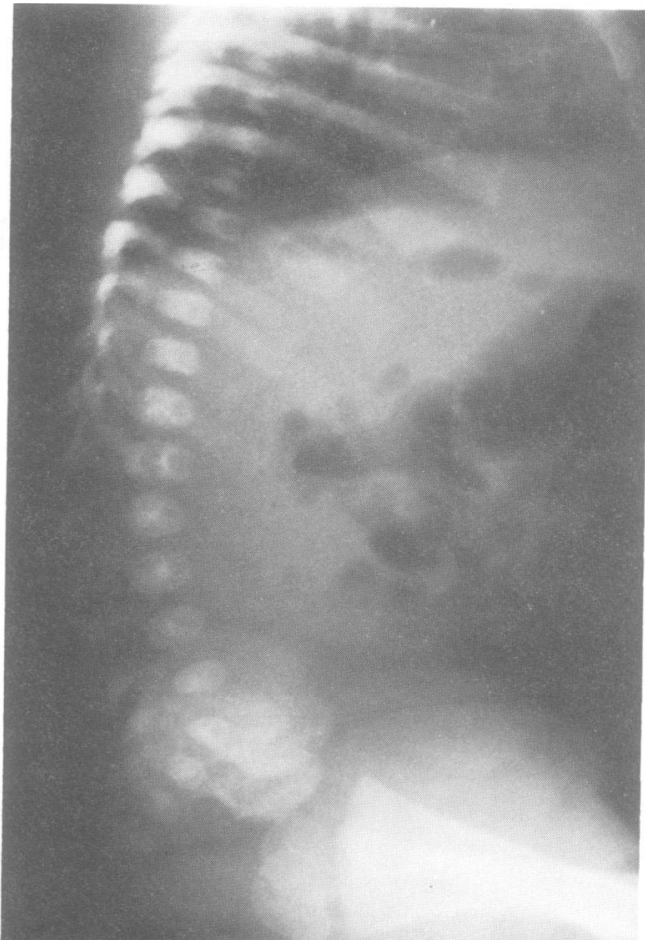

Figure 2 Lateral radiograph of the trunk at 1 week of age showing ovoid lumbar vertebral bodies.

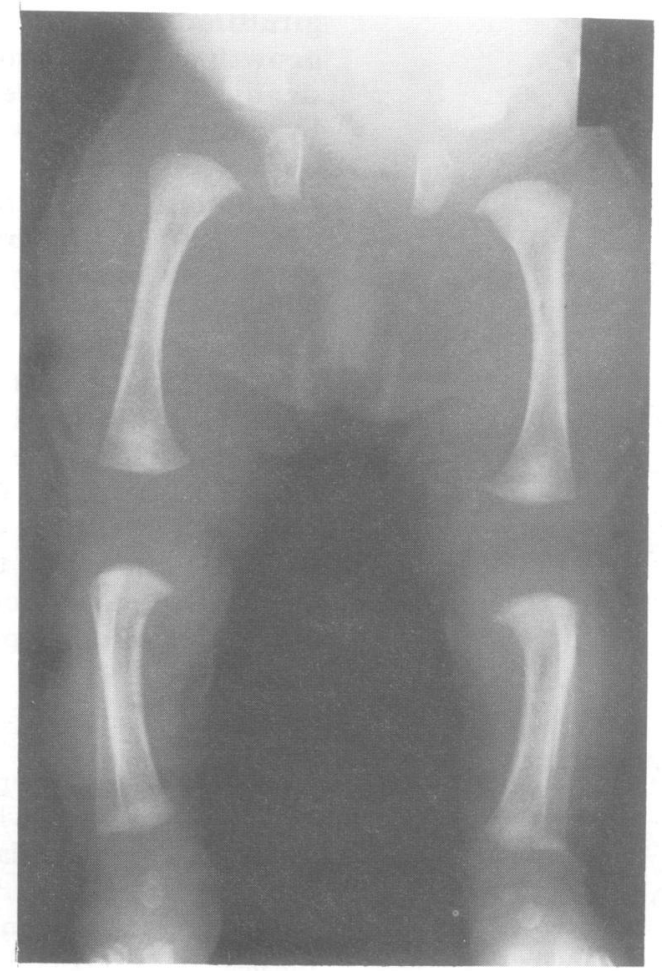

Figure 3 Radiograph of the legs at 1 week of age showing absence of ossification in the femoral and tibial epiphyses, flaring of their metaphyses, and absent ossification of the pubic symphysis.

in the lumbar spine. The calvarium was large, elongated posteriorly, and had wide open sutures. The floor of the anterior fossa was steep. The facial bones and the mandible were hypoplastic.

Her longitudinal growth remained well below the normal range but it followed at or a little below the mean growth curve for children with spondyloepiphyseal dysplasia congenita (fig 4). ${ }^{12}$ When last reviewed at the age 


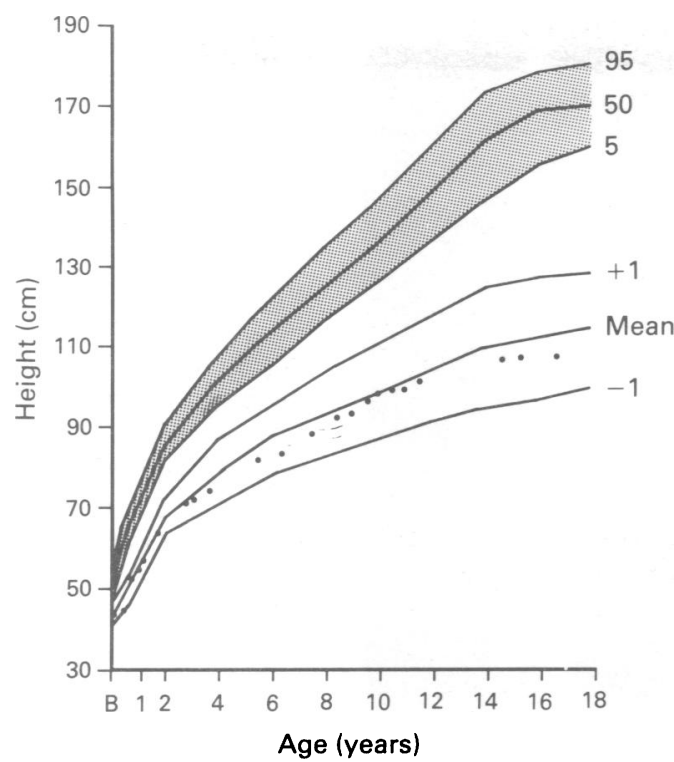

Figure 4 Growth pattern from birth to 17 years of age. The normal centile growth curves are shaded. The lower curves, including the mean and $1 S D$ on either side of the mean, were based on the growth of 62 males and females with spondylepiphyseal dysplasia congenita. ${ }^{22}$ The growth of our patient is shown at or a little below the mean curve for patients with this disorder.

of 17 years, her height was $108 \mathrm{~cm}$ and her weight was $30 \cdot 3 \mathrm{~kg}$.

After repair of her cleft palate at 1 year of age, her speech developed normally with excellent velopharyngeal function. Her cognitive development was normal but her motor development was delayed because of hypotonia and the skeletal anomalies. She started standing at 18 months of age and walking at 19 months of age.

She had an outset gait owing to an excessive range of external rotation and lack of internal rotation of the hips. Flexion and extension of the hips were normal in early childhood but mild fixed flexion deformities of the hips developed in later childhood. Coxa vara resulted in increased adduction and reduced abduction of the hips. The range of abduction of the hips diminished during growth owing to progressive coxa vara, short and broad femoral necks, as well as impingement of the greater trochanters on the pelvis.

By 3 years of age, she had developed a small triangular fragment in the lower metaphysis of each femoral neck (fig 5). These anomalies were probably the result of mild slippages of the proximal femoral epiphyses as a result of shear forces on the abnormally vertical physes. ${ }^{13}$ At the age of 12 years, bilateral Pauwel's intertrochanteric osteotomies of the femora and arrests of the greater trochanteric physes were undertaken because of increasing coxa vara with worsening Trendelenberg gait (fig 6) ${ }^{13}$ In each hip, the neck-shaft angle and the plane of the proximal femoral physes were restored to normal. The metaphyseal fragments healed and the greater trochanters no longer impinged on the pelvis (fig 7). She still had a mild limp but her physical endurance improved markedly. Ossification centres had not appeared in either proximal femoral epiphysis by 17 years of age. MRI scans of the hips showed that the cartilaginous proximal femoral epiphyses were flatter and broader than normal but the articular surface appeared congruent with the acetabulum (fig 8).

Her knees were normally aligned at birth but by 2 years of age she had bilateral genu valgum, worse on the left side (fig 5). The severity of the genu valgum increased minimally during growth. The patellofemoral joints were stable but the patellae were located further laterally than normal. She maintained a full range of flexion but an increasing range of hyperextension of each knee during growth. At 17 years of age, her right knee was noted to clunk and give way, which was probably because of cruciate ligament laxity.

Delayed ossification of the distal femoral and proximal tibial epiphyses was observed on
Figure 5 Radiograph of the legs at 3 years of age showing coxa vara, absence of ossification in the proximal femoral epiphyses, small and abnormally shaped ossific nuclei of the other epiphyses, and genu valgum.

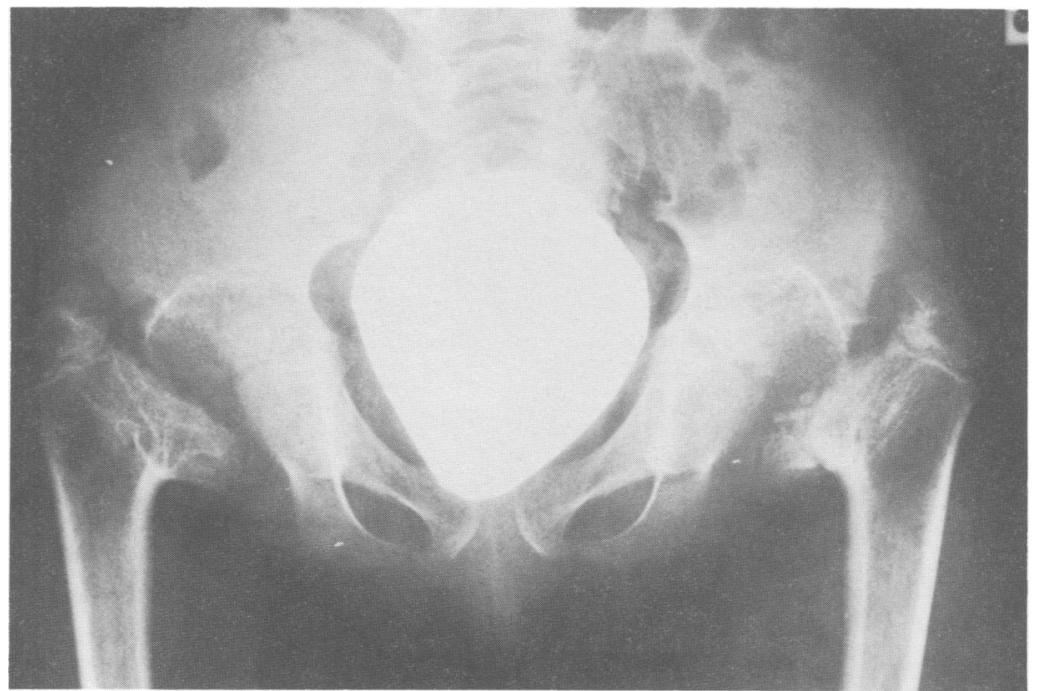

Figure 6 Radiograph of the pelvis at 12 years of age. Each hip shows coxa vara short broad femoral neck, high greater trochanter, lack of ossification of the femoral epiphysis, a metaphyseal fragment, and abnormal acetabular development. 


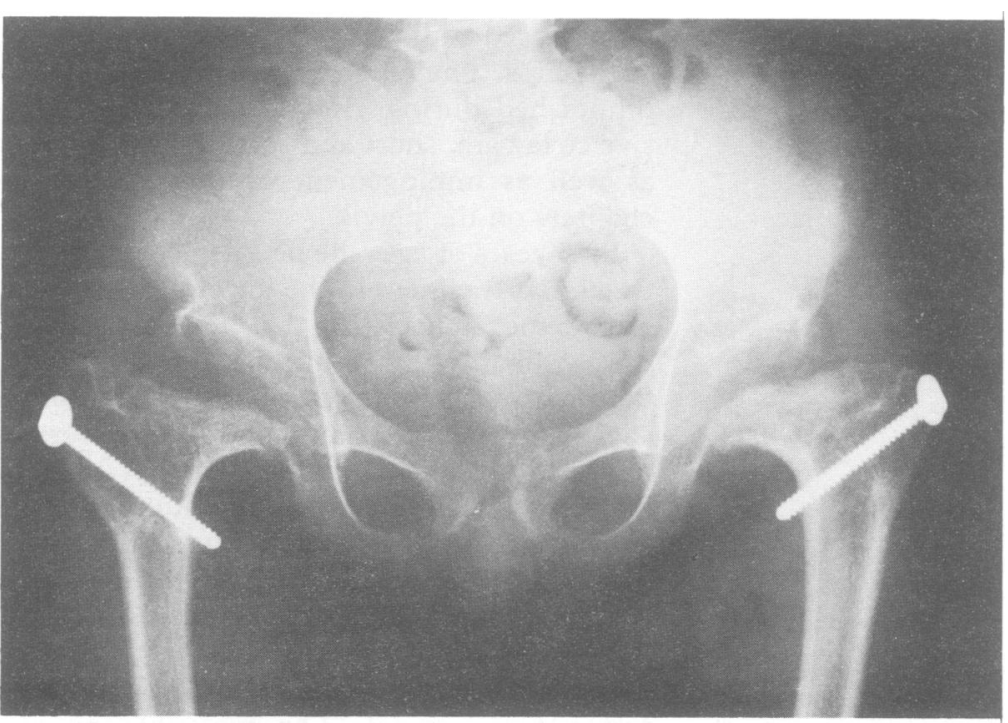

Figure 7 Radiograph of the pelvis at 14 years of age. The appearance of the hips has improved after bilateral realignment osteotomies of the proximal femora and closure of the physes of the greater trochanters.

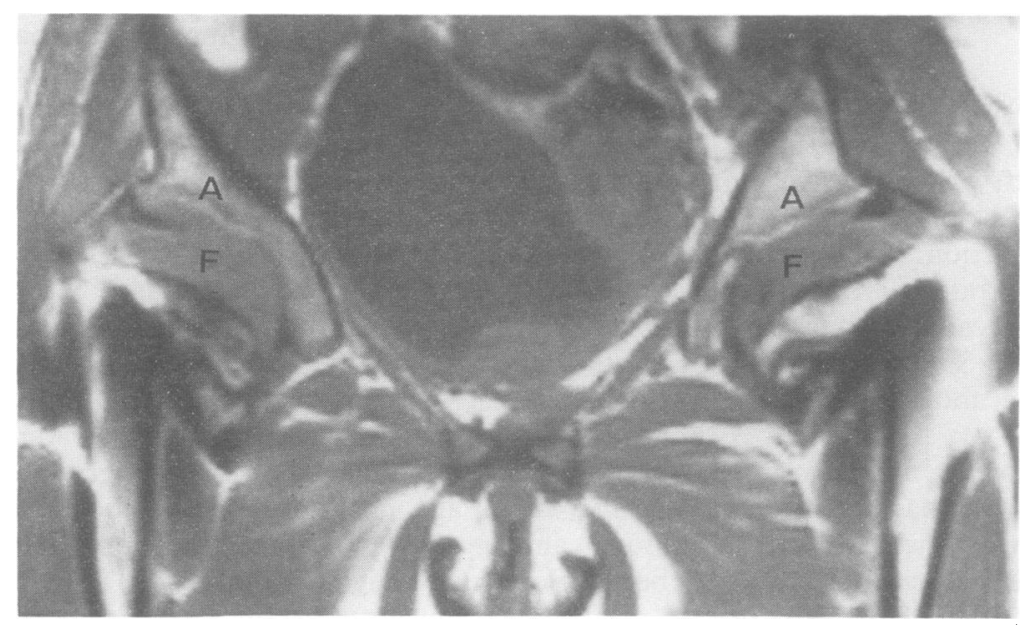

Figure 8 Coronal MRI scan of the pelvis at 17 years of age. The unossified proximal femoral epiphyses $(F)$ are flat but with good acetabular coverage $(A)$. The screws in the femoral necks have produced some artefacts.

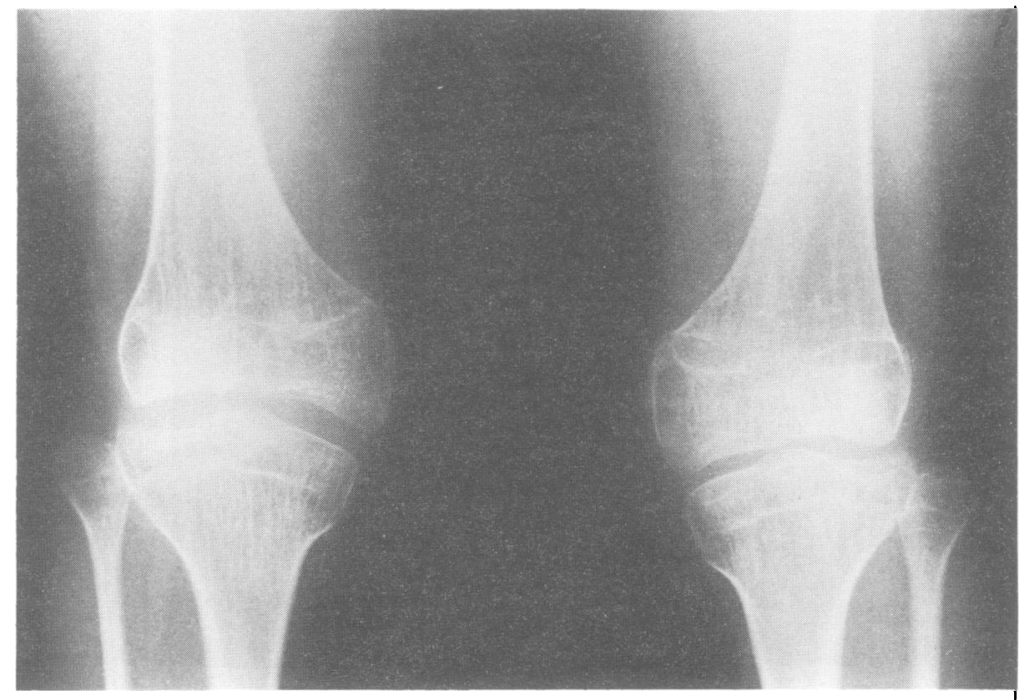

Figure 9 Radiographs of the knees at 15 years of age. The physes have closed and the femoral and tibial condyles are misshapen and appear incongruent. serial radiographs. At all ages, the ossification centres were smaller than normal and abnormally shaped (fig 5). After closure of the physes, the femoral and tibial condyles appeared to be incongruous (fig 9). However, MRI scans showed that the articular surfaces were congruent as an abnormal amount of unossified cartilage covered the condyles, particularly the medial femoral condyles (figs 10 and 11). The menisci and cruciate ligaments appeared normal.

Ossification of the distal tibial epiphyses was delayed but her ankle shape and range of movement were normal (figs 3 and 5). She had mobile pes planovalgus which exaggerated her outset gait.

Her upper limbs were well aligned and all joints had a normal range of movement. Serial radiographs showed that the ossification centres of all epiphyses were slow in appearing, small, and abnormally shaped.

Her neck was short but had a full range of movement. There was generalised platyspondyly of the cervical vertebrae and elongation of the pedicles. The odontoid process of the axis was underdeveloped but she did not develop any clinical evidence of spinal cord compression. At 4 years of age, standard tomograms showed hypoplasia of the tip of the odontoid process (fig 12). On flexion of the neck, the anterior arch of the atlas moved minimally forward. However, on extension of the neck it moved posteriorly to lie on top of the odontoid

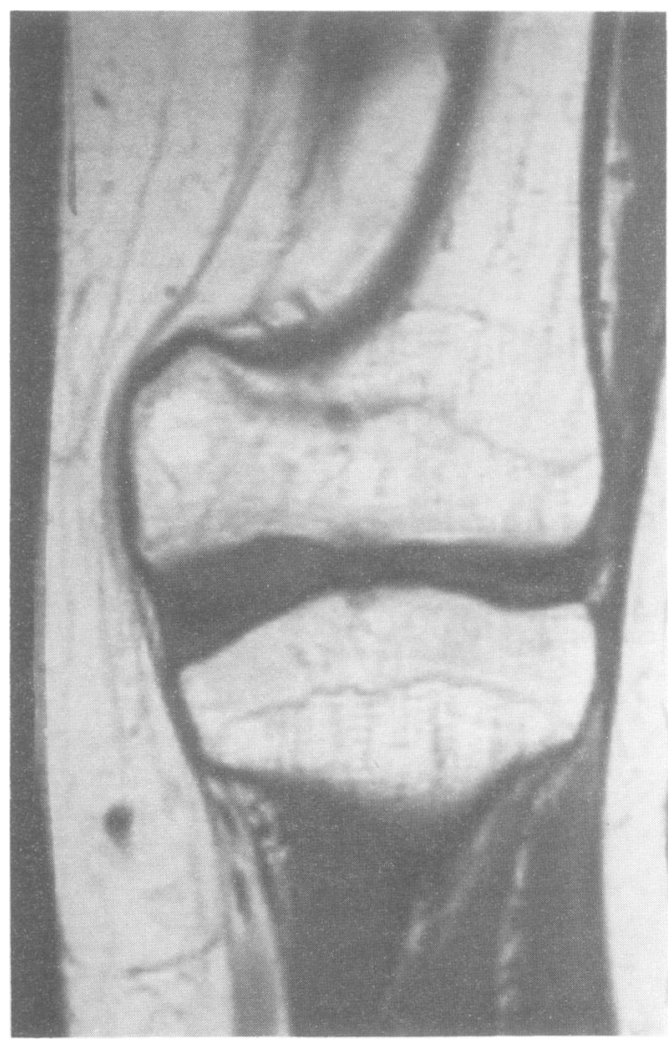

Figure 10 Coronal MRI of the left knee at 17 years of age showing that the articular surfaces are more normal in shape and congruency than expected from the plain radiographs. There is a thick layer of unossified cartilage on the medial femoral condyle. The menisci appear normal. 


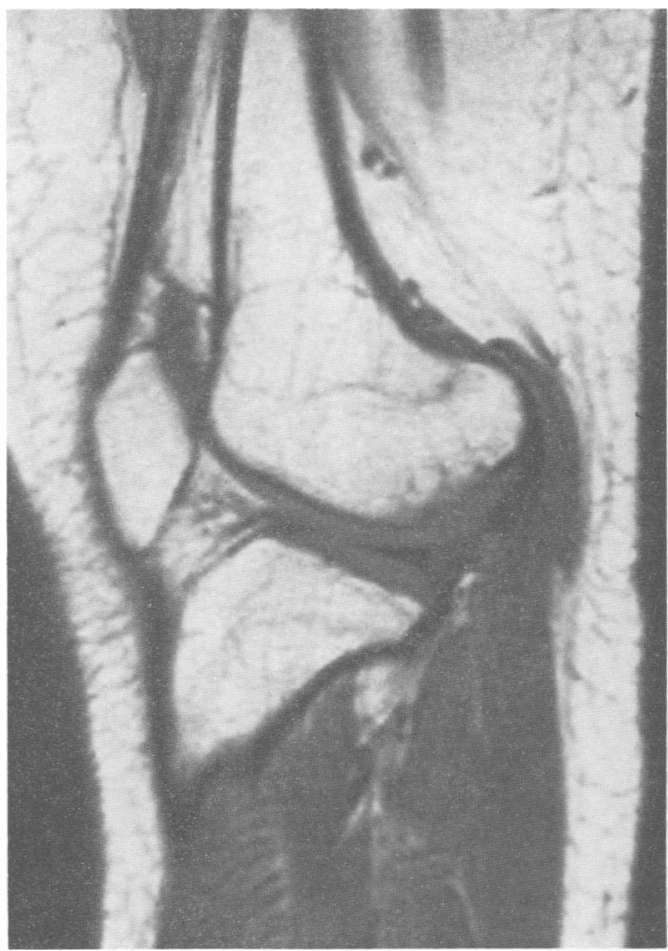

Figure 11 Sagittal MRI of the left knee at 17 years of age showing irregular ossification of the posterior portion of the femoral condyle, thick articular cartilage, and an apparently congruent joint.

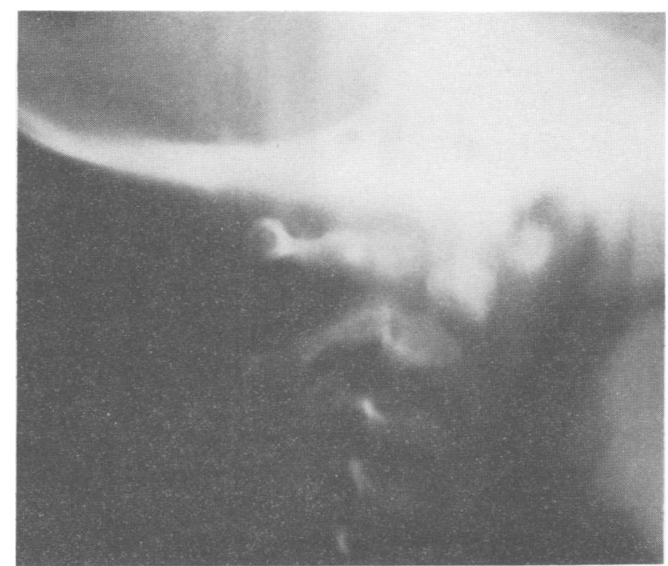

Figure 12 Lateral tomogram of the upper cervical spine with the neck in flexion at 4 years of age. The odontoid process of the axis is hypoplastic.

process. Repeat standard tomograms and computerised tomograms at later ages showed minimal anterior and posterior movement of the anterior arch of the atlas. The summit ossification centre which normally appears at 3 to 6 years of age did not appear. ${ }^{14}$ The synchondrosis between the odontoid process and neural arches and the body fused between 5 and 6 years, the normal age of fusion. ${ }^{14}$ An MRI scan of the neck at 17 years of age confirmed hypoplasia of the tip of the odontoid process, fusion of the synchondroses at the base of the odontoid process, and absence of impingement of the odontoid process on the spinal cord or brain stem (fig 13). The brain stem, mid brain, cerebellum, and occipital lobes appeared normal on MRI scan.

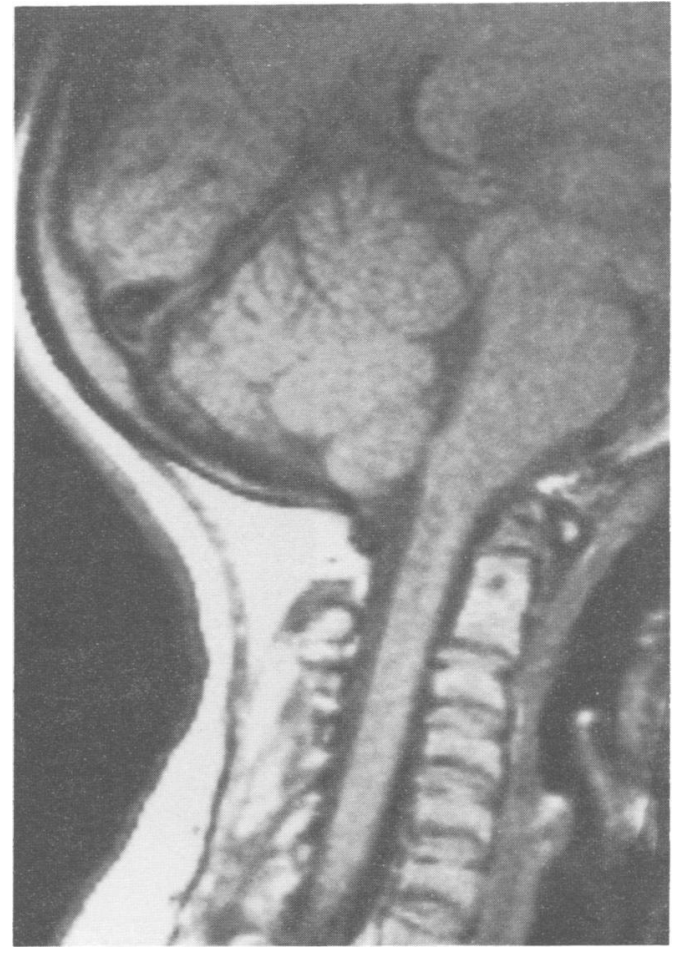

Figure 13 Sagittal MRI scan of the upper cervical spine and posterior fossa at 17 years of age. There is generalised platyspondyly and hypoplasia of the tip of the odontoid process. The spinal cord, brain stem, and cerebellum are normal.

The thoracolumbar spine was normally aligned at birth (fig 1). After starting standing and walking, she rapidly developed a more protuberant abdomen, excessive lumbosacral lordosis, thoracic kyphosis, pectus carinatum, and a more obvious barrel chest. A rigid right thoracolumbar scoliosis also developed at approximately 2 years of age. It showed minimal progression. These early changes in spinal contour and length altered her body proportions to produce a disproportionately short trunk form of dwarfism (figs 14 and 15).

Serial radiographs confirmed the clinical changes in spinal contour. Platyspondyly and irregular vertebral shape worsened with growth (figs 16 and 17). In the lumbar spine, she developed dorsal wedging of the vertebral bodies and elongation of the pedicles. The thoracic vertebral bodies were markedly flattened and misshapen by late childhood. Widespread secondary osteoarthritis was evident in the early teens.

Yearly examinations of vision and of the anterior and posterior chambers of the eyes showed moderately severe myopia but no other anomalies.

Hearing impairment was suspected when she was 3 years of age. Audiometry showed she had right conductive loss for the low tones owing to fluid within the middle ear following repeated upper respiratory tract infections. This loss improved after myringotomy and drainage of the middle ear. Her left ear drum had an abnormal impedence consistent with a hypermobile drum. At 17 years of age, the impedence of the left ear drum was still abnormal but less mobile than previously. She also 


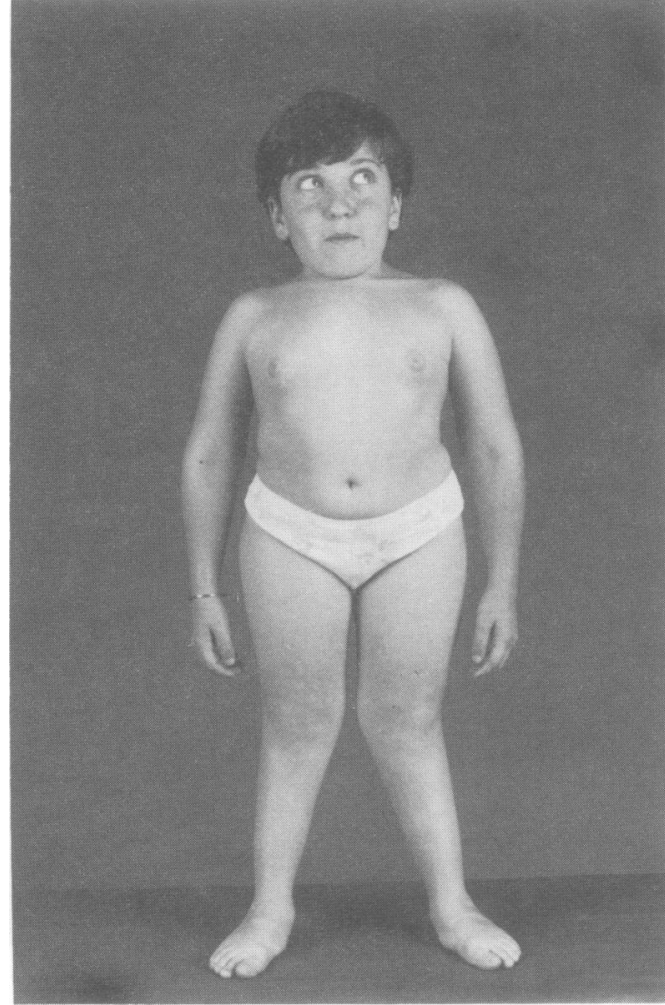

Figure 14 Appearance at 11 years of age showing shor limbs, genu valgum, pes planovalgus, and disproportionately short trunk.

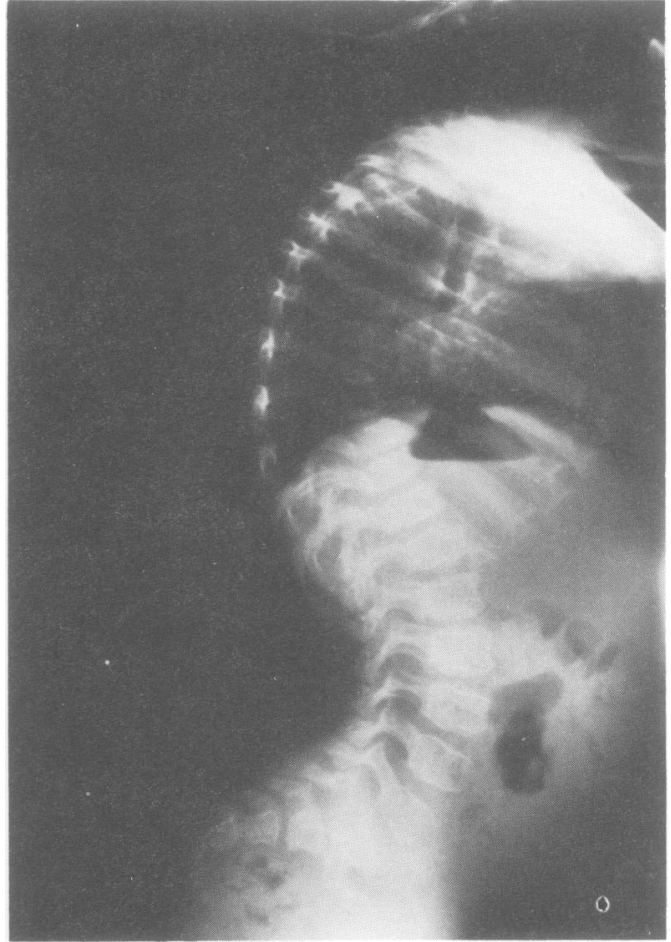

Figure 16 Lateral radiograph of the spine at 3 years of age showing excessive lumbar lordosis and thoracic kyphosis. There is widespread platyspondyly with elongation of the pedicles of the lumbar vertebrae.

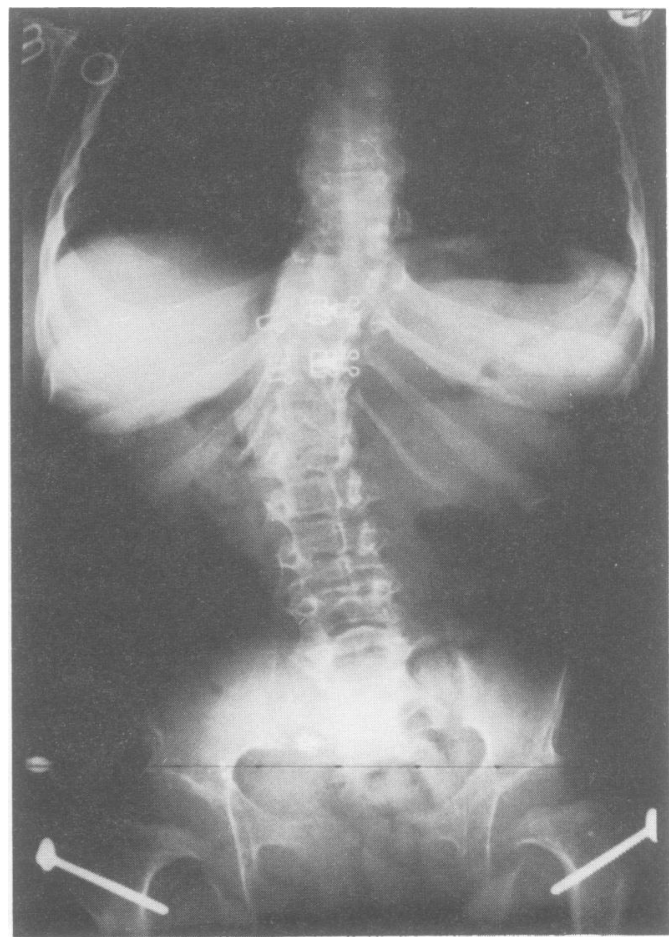

Figure 17 Radiograph of the spine at 14 years of age showing a structural right thoracolumbar scoliosis, generalised platyspondyly, and a barrel shaped thorax.

had bilateral, moderately severe high frequency hearing loss at $4000 \mathrm{~Hz}$. Repeat audiometry at 14 and 17 years of age confirmed the earlier findings of sensorineural hearing loss at high frequencies. The loss was greater than at 3 years of age. At 17 years of age, she had a $50 \mathrm{db}$ hearing loss in both ears at $4000 \mathrm{~Hz}$ and
Figure 15 Appearance at 11 years of age showing short neck and trunk, excessive lumbar lordosis with protuberant abdomen, and thoracic kyphosis with prominence of the sternum and anterior chest wall. 
a $70 \mathrm{db}$ loss at $8000 \mathrm{~Hz}$. Anti-type II collagen antibodies, which have been implicated in the pathogenesis of sensorineural deafness in patients with rheumatoid arthritis, were not detected in her serum..$^{15}$ Her vestibular function was not determined.

At 8 years of age, her dental development was clinically normal except that only the maxillary left first permanent molar had erupted. Panoramic radiographs at 8 years and 17 years of age confirmed normal development of the maxillary left first permanent molar but the other first permanent molars were either congenitally absent or severely delayed in their development. The radiographs also showed that the maxilla and mandible were a little smaller than normal although cephalometric measurements were not undertaken.

\section{Discussion}

This patient, with a mutation of type II collagen, had the typical clinical and radiological features of spondyloepiphyseal dysplasia congenita with major anomalies of the peripheral and axial skeleton and of the craniofacial structures. The tissues involved were those that were expected from the known mammalian patterns of expression of the COL2A1 gene by chondrogenic and non-chondrogenic cells. ${ }^{116}$

Although we were unable to study the type II collagen protein in our patient, because cartilage was unavailable, it is likely that a mixture of normal and mutant type II collagen molecules were present in the involved tissues. This prediction was based on the protein findings in patients who were heterozygous for substitutions of glycine 943 by serine or arginine 519 by cysteine. ${ }^{1718}$ We expect that a mixture of normal and mutant $\alpha 1$ (II) chains was produced by chondrocytes and other cell types that expressed the COL2A1 gene. Approximately $75 \%$ of the type II collagen molecules would be heterotrimers containing a mixture of normal and mutant $\alpha 1$ (II) chains, $12.5 \%$ would be mutant homotrimers containing only mutant chains, and $12.5 \%$ would be normal homotrimers. The substitution of glycine 997 by serine interrupts the mandatory Gly-X-Y triplet structure of the helical domain of the $\alpha$ l(II) chain so that helix formation and stability would be abnormal in the heterotrimers and mutant homotrimers. ${ }^{17}$ Such molecules would be enzymatically overmodified, unstable, and poorly secreted. As a result, it is likely that the involved tissues would contain some mutant type II collagen and a grossly reduced amount of normal type II collagen.

Her short stature was the result of poor longitudinal growth and deformities of her long bones and vertebrae. Serial radiographs showed generalised abnormalities of the physeal and epiphyseal cartilages and the intervertebral discs. Even at 17 years of age, the ossific nuclei of the femoral heads had not appeared and ossification of the femoral condyles was incomplete although the articular surfaces appeared congruent.

Asymmetrical growth of the proximal femoral physes and the greater trochanteric apophyses resulted in progressive coxa vara that required surgical correction. Asymmetrical growth of the distal femoral physes and epiphyses produced mild genu valgum which increased slowly throughout childhood. Asymmetrical growth of the lumbar vertebrae was also observed with elongation of the pedicles and wedging of the vertebral bodies. Abnormal ossification of the tip of the odontoid process was associated with mild instability between the atlas and axis that did not warrant atlantoaxial fusion.

Complex developmental abnormalities of the craniofacial structures were also observed. Abnormal endochondral ossification would account for the smallness of the skull base and face. Early mandibular hypoplasia, owing to abnormal Meckel's cartilages, with consequent extrinsic obstruction of palatal closure by a posteriorly displaced tongue would also account for the U shaped cleft palate. ${ }^{1619}$ Type II collagen cannot be directly implicated in the production of the palatal defect as the developing palate lacks this collagen. ${ }^{20}$

The asymmetrical developmental anomalies of the teeth, involving three of the first permanent molars which were either absent or severely delayed in appearance, are unusual in spondyloepiphyseal dysplasia congenita. ${ }^{21}$

The moderately severe myopia can be explained by the adverse effects of the type II collagen mutation, expressed by non-chondrogenic cells, on eye development and structure. Type II collagen appears transiently beneath the surface ectoderm of the optic vesicle and in the neural retina, the corneal and conjunctival epithelia, and the sclera of the developing mammalian eye. ${ }^{116}$ Type II collagen in the vitreous humour of the differentiated eye is found, with type IX collagen, in a network of 7 to $13 \mathrm{~nm}$ diameter fibres embedded in a hyaluronate gel. ${ }^{22}$ Abnormalities of this network contribute to the production of retinal detachment in patients with spondyloepiphyseal dysplasia congenita. ${ }^{21}$

The middle and inner ear abnormalities can also be explained by the adverse effects of the mutation on the development and structure of the ear. During normal development of the mammalian ear, type II collagen is found first beneath the otic vesicle epithelium and the adjoining neuroepithelium. ${ }^{16}$ With further differentiation, type II collagen is observed on the basal and apical surfaces of the epithelium of most regions of the inner ear, including the cochlea, and in the cartilaginous periotic mesenchyme. ${ }^{16}$ In the differentiated inner ear, type II collagen is a major fibrous component of the osseous spiral lamina, spiral limbus, and tectorial membrane. ${ }^{2324}$ Abnormalities of these structures, particularly in the basal turn of the cochlea, provide an explanation for her severe high frequency sensorineural hearing loss. ${ }^{25}$

Abnormal formation of the cartilaginous eustachian tubes may have contributed to the middle ear deafness that was associated with repeated middle ear infections in early life. Abnormal development of the ossicular chain, which is also formed from cartilage, was probably responsible for the hypermobility of 
one of her ear drums. The malleus and incus are derived from the first branchial arch cartilage and the stapes from the second branchial arch cartilage. ${ }^{23}$

Wood et $a l^{16}$ have proposed that the type II collagen produced transiently by epithelial cells of the developing head and neck may act as a morphogenetic signal that specifies the form of the vertebrate chondrocranium. Comparison of the three dimensional distribution of the early appearing type II collagen and the subsequent shape of the chondrocranial cartilages indicates that the chondrocranial form may be derived from a 'prepattern' of epithelially derived type II collagen expressed at epithelial-mesenchymal tissue interfaces. ${ }^{16}$

We were unable to find any clinical anomalies in other regions in which type II collagen has been found. It is transiently expressed during the development of specific regions of the brain and cervical spinal cord, heart, epidermis, tendon, and calvarial mesenchyme. ${ }^{116}$ An MRI scan of the cervical spinal cord, mid brain, cerebellum, and occipital lobe of the cerebrum did not show any anomaly. Type II collagen is a major component of the hyaline cartilages of the larynx and tracheobronchial tree but there were no clinical anomalies of these structures.

The clinical features of our patient were similar to those reported in two other children who also had heterozygous mutations involving exon 48 of the COL2A1 gene. ${ }^{34}$ This observation and the reported milder phenotype produced by a heterozygous substitution of arginine 519 by cysteine suggests that carboxy-terminal mutations of the triple helix may produce severer phenotypes than more amino-terminal mutations. ${ }^{5}$ Such a proposal is in keeping with the findings in osteogenesis imperfecta. ${ }^{26}$ However, the achondrogenesishypochondrogenesis phenotype produced by a heterozygous point mutation in exon 46 leading to the substitution of glycine 943 by serine is severer than the phenotypes produced by the three reported mutations that involve exon $48 .^{6}$ As in osteogenesis imperfecta, it is to be expected that such exceptions will occur as not only the type of mutation, but also its site, surrounding sequences, and other unknown genetic and epigenetic factors are likely to influence the phenotype.

Transgenic mouse studies have confirmed that anomalies of type II collagen can result in phenotypes similar to achondrogenesis-hypochondrogenesis and spondyloepiphyseal dysplasia congenita. In one of these models, a construct containing a diphtheria toxin A chain gene under the control of the rat COL2A1 collagen promoter and enhancer produced fetuses that were small with short limbs and a cleft palate. ${ }^{27}$ The DNA constructs were designed to ablate chondrocytes in order to study the regulation and role of type II collagen in embryogenesis. In another model, expression of a DNA construct consisting of a minigene version of the human COL $2 \mathrm{Al}$ gene produced a form of spondyloepiphyseal dysplasia with dwarfism, short and thick limbs, a short snout, a cranial bulge, a wide cleft palate, and delayed ossification of epiphyses. ${ }^{28}$ The DNA construct contained a large in frame deletion that produced shortened $\alpha 1$ (II) chains capable of disulphide bonding to form mutant homotrimers but also disulphide bonding with normal $\alpha 1(\mathrm{II})$ chains to produce heterotrimers. The latter molecules, because of the disparity in the normal and mutant chain lengths, were probably degraded. Finally, transgenic mice expressing introduced mutations that resulted in the substitution of glycine 85 by cysteine, the cross linking lysine 87 of the helix by arginine, the cross linking lysine residue of the aminotelopeptide by arginine, or deletion of exon 7, which removed residues 4 to 18 of the triple helix, resulted in many neonatal deaths and a few surviving pups. ${ }^{29}$ Affected mice were dwarfed with short limbs and trunk, craniofacial deformities, and cleft palate. Neonatal death was from acute respiratory distress caused by an inability to inflate the lungs at birth. In all of these transgenic mouse models, the severity of the phenotype appeared to be dependent on the level of expression of the transgene.

The findings in the present study confirm the importance of heterozygous mutations of type II collagen in producing the spondyloepiphyseal dysplasia congenita phenotype. Further mutations need to be characterised in order to understand better the pathogenesis of the various phenotypes within the chondrodysplasia family of type II collagenopathies.

The authors express their appreciation to $\mathrm{V}$ Mayne for her radiological advice, to E Kier for his audiometry studies, and to $M$ Rowley for her measurements of serum anti-type II collagen antibodies. The work by W G Cole was supported by the National Health and Medical Research Council of Australia and the Royal Children's Hospital Research Foundation, Melbourne.

1 Cheah KSE, Lau ET, Au PKC, Tam PPL. Expression of the mouse $\alpha$ l(II) collagen gene is not restricted to cartilage during development. Development 1991;111:945-53.

2 Spranger J. Bone dysplasia 'families'. Pathol Immunopathol Res 1988;7:76-80.

3 Lee B, Vissing H, Ramirez F, Rogers D, Rimoin D. Identification of the molecular defect in a family with spondyloepiphyseal dysplasia. Science 1989;244:978-80.

4 Tiller GE, Rimoin DL, Murray LW, Cohn DH. Tandem duplication within a type II collagen gene (COL2A1) exon in an individual with spondyloepiphyseal dysplasia. Proc Natl Acad Sci USA 1990;87:3889-93.

5 Ala-Kokko L, Baldwin CT, Moskowitz RW, Prockop K. Single base mutation in the type II procollagen gene (COL2A1) as a cause of primary osteoarthritis associated with a mild chondrodysplasia. Proc Natl Acad Sci USA with a mild cho

6 Vissing H, D'Alessio MD, Lee B, Ramirez F, Godfrey M, Hollister DW. Glycine to serine substitution in the triple helical domain of pro- $\alpha 1$ (II) collagen results in a lethal perinatal form of

7 Francomano CA, Liberfarb RM, Hirose T, et al. The Stickler syndrome: evidence for close linkage to the structural gene for type II collagen. Genomics 1987;1:2936.

8 Ahmad NH, Ala-Kokko L, Knowlton G, et al. Stop codon in the procollagen II gene (COL2A1) in a family with the Stickler syndrome (arthro-ophthalmology). Proc Nat Acad Sci USA 1991;88:6624-7.

9 Poole AR, Pidoux I, Reiner A, et al. Kniest dysplasia is characterized by an apparent abnormal processing of the c-propeptide of type II cartilage resulting in imperfect c-propeptide of type II cartilage resulting in

10 Chan D, Cole WG. Low basal transcription of genes for tissue-specific collagens by fibroblasts and lymphoblastoid cells. I Biol Chem 1991;266:12487-94. 
11 Kitchen WH, Robinson HP, Dickinson AJ. Revised intrauterine growth curves for an Australian hospital population. Aust Paediatr 7 1983;19:157-61.

12 Horton WA, Hall JG, Scott CI, Pyeritz RE, Rimoin DL. Growth curves for height for diastrophic dysplasia, spondyloepiphyseal dysplasia congenita, and pseudoachondroplasia. Am f Dis Child 1982;136:316-19.

13 Cordes S, Dickens DRV, Cole WG. Long-term correction of coxa vara in childhood by the Pauwels Y-shaped osteotomy. F Bone foint Surg (Br) 1991;73:3-6.

14 Bailey DK. The normal cervical spine in infants and children. Radiology 1952;59:712-19.

15 Clague RB, Firth SA, Holt PJL, et al. Serum autoantibodies to type II collagen in rheumatoid arthritis: comparison of six immunological methods and clinical features. Ann Rheum Dis 1983;42:537-44.

16 Wood A, Ashhurst DE, Corbett A, Thorogood P. The transient expression of type II collagen at tissue interfaces during mammalian craniofacial development. Development 1991;111:955-68.

17 Godfrey M, Hollister DW. Type II achondrogenesis-hypochondrogenesis: identification of abnormal type II collagen. Am f Hum Genet 1988;43:904-13.

18 Eyre DR, Weis MA, Moskowitz RW. Cartilage expression of a type II collagen mutation in an inherited form of osteoarthritis associated with a mild chondrodysplasia. $f$ Clin Invest 1991;87:357-61.

19 Carey JC, Fineman RM, Ziter FA. The Robin sequence as a consequence of malformation, dysplasia, and neuromuscular syndromes. $\mathcal{f}$ Pediatr 1982;101:858-64.

20 Ferguson MWJ. Palate development. Development 1988;103:41-60.
21 Gorlin RJ, Cohen MM Jr, Levin LS. Syndromes of the head and neck. New York: Oxford University Press, 1990: 2213.

22 Bailey AJ. Structure, function and ageing of the collagens of the eye. Eye 1987;1:175-83.

23 Yoo TJ, Floyd R, Ishibe T, Shea JJ, Bowman C. Immunological testing of certain ear diseases. Am 7 Otol 1985;6:96-100.

24 Slepecky NB, Savage JE, Cefaratti LK, Yoo TJ. Electronmicroscopic localization of type II, IX, and V collagen in the organ of Corti of the gerbil. Cell Tissue Res 1992;267:413-18.

25 Steele CR. Cochlear mechanics. In: Keidel WD, Neff WD, eds. Handbook of sensory physiology. Vol 3. Auditory system. Clinical and special topics. Berlin: Springer-Verlag, 1976: 444-78.

26 Cole WG, Chow CW, Rogers JG, Bateman JF. The clinical features of three babies with osteogenesis imperfecta resulting from the substitution of glycine by arginine in the pro $\alpha 1(\mathrm{I})$ chain of type I procollagen. $\mathcal{F}$ Med Genet 1990;27:228-35.

27 Bruggeman LA, Xiang XH, Brown KS, Yamada Y. Developmental regulation for collagen II gene expression in transgenic mice. Teratology $1991 ; 44: 203-8$.

28 Vandenberg $P$, Khillan JS, Prockop DJ, Helminen $H$, Kontusaari S, Ala-Kokko A. Expression of a partially type II procollagen (COL2A1) in transgenic mice produces a chondrodysplasia. Proc Natl 29 Acad Sci USA 1991;88:7640-4.

Garofalo S, Vuorio E, Metsaranta M, et al. Reduced amounts of cartilage collagen fibrils and growth plate anomalies in transgenic mice harboring a glycine-tocysteine mutation in the mouse type II procollagen $\alpha 1-$
chain gene. Proc Natl Acad Sci USA 1991;88:9648-52. 